\title{
Practical problem-based programming learning using video
}

\author{
Shigeo Tsukuta ${ }^{1} \cdot$ Takashi Yamaguchi $^{1} \cdot$ Tatsuya Kano $^{1} \cdot$ Yorinori Kishimoto $^{1} \cdot$ Eiji Nunohiro $^{1}$
}

Received: 10 April 2018 / Accepted: 18 February 2019 / Published online: 12 April 2019

(c) The Author(s) 2019

\begin{abstract}
The fundamental skills for information system development such as system designing, programming and project management are very similar to the fundamentals of general problem solving. In this paper, we proposed an education framework for practical problem solving based on system designing technologies and an application of proposed framework on video training materials to train the skills of modeling and understanding from ambiguous matter in practical problem via nonverbalized video training material. Our framework uses Resource Flow Diagram (RFD) to support the understanding of procedure and resources on problem solving. RFD is our proposed visualization method for procedure and resource management based on Sequence Diagram in Unified Modeling Language (UML). RFD is designed for the intuitive representation of the procedure flow and required resources since UML could not define them with single diagram. In this experiment, proposed education framework was applied for the understanding of cooking procedure from the cooking exhibition videos on the demonstrative lecture.
\end{abstract}

Keywords E-learning $\cdot$ System design $\cdot$ Requirement definition $\cdot$ Functional programming $\cdot$ Education by video

\section{Introduction}

Recently, the education of highly skilled human resource is very important because the need for advanced knowledge and skills has grown due to the progression of networked information society. The fundamental skills for information system development such as system designing, programming and project management are very similar to the fundamentals of general problem solving. Therefore, the methods and applications of the elementary education for informatics are discussed in Japan.

In this paper, we proposed an education framework for practical problem solving based on system designing technologies and an application of proposed framework on video training materials to train the skills of modeling and understanding from ambiguous matter in practical problem via non-verbalized video training material. Our framework uses Resource Flow Diagram (RFD) to support the understanding

This work was presented in part at the 23rd International Symposium on Artificial Life and Robotics, Beppu, Oita, January 18-20, 2018.

Shigeo Tsukuta

shigeo@tsukuta.com

1 Tokyo University of Information Sciences, Chiba, Japan of procedure and resources on problem solving. RFD is our proposed visualization method for procedure and resource management based on Sequence Diagram in UML $[1,2]$.

In this experiment, proposed education framework is applied for the understanding of cooking procedure from exhibition video of cooking. Materials and main tools and procedures are shown in the video of cooking. The important viewpoint of actual cooking problem is resource management that is to avoid the confliction of infrastructure such as the cooking table or the gas range in actually cooking; however, it is not shown in exhibition video. In addition, tools such as bowls for putting cut materials may not be described in the recipe may be necessary in some cases. The learners have to clarify the cooking procedures and resources from ambiguous video information same as other problem in practical life.

\section{Methods}

\subsection{Education of modeling from video}

The various events such as general problems for peoples in real world can be modeled by 3 elements of Procedure, Resources and Tools such as boiling, meat and water, and 
pan and stove in the cooking, and method, input and output, and program library in the programing. In this research, we focus on extracting these elements from video to improve problem-solving skill that can be applied to the information system design, development, and programing.

The elements extraction from video is different from extraction from sentences in earlier research examined by Nunohiro et al. [3]. The video contents are complex and similar to practical problems; therefore, it is effective to training problem-solving skill for practical problems. Generally, the video teaching materials produce intuitive understanding to student; however, the perspicuity depends on the quality of video contents. On the other hand, the quality requirement of video teaching material in our education model is low

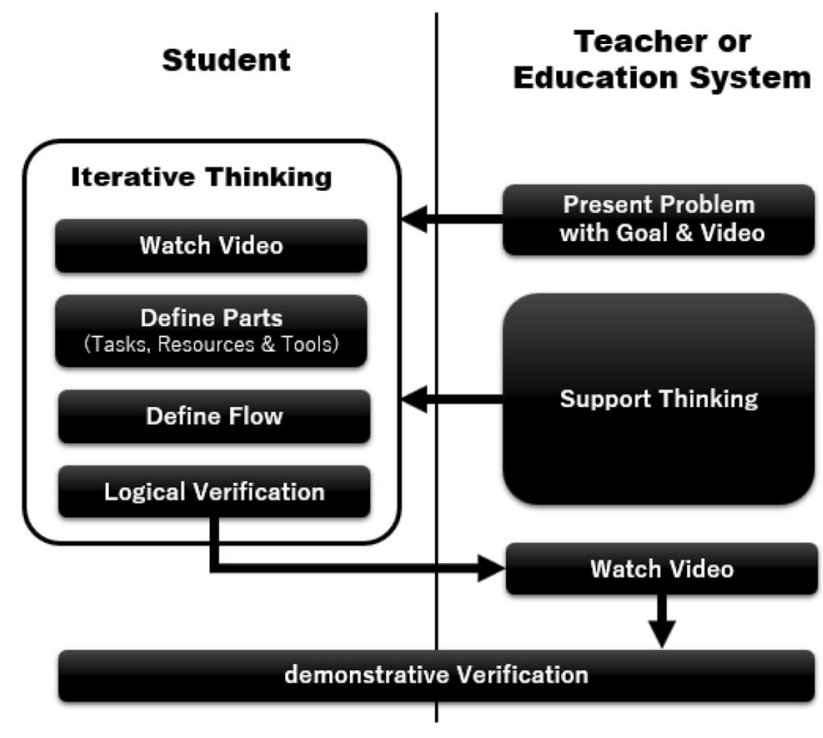

Fig. 1 Overview of our education model using video compared with general education model using video because our aim is the modeling of events from ambiguous things.

The overview of education is shown in Fig. 1. Our problem-solving framework is based on practice centered. First, teacher or education system present the goal of problem with video. After this, student work their self with iterative thinking of video watching, parts definition such as resource and tools, flow definition and logical verification by own self. The teacher or education system support this iterative thinking. Finally, it is desirable that verification of own-solution is verified in demonstration.

In this education flow, most important problem is how to support iterative thinking because these discussions about event modeling require high linguistic competence to student and teacher. In this paper, we investigate the nonlinguistic dependency support tool for iterative thinking based on information system designing technologies.

\subsection{Resource Flow Diagram}

In this paper, we proposed a Resource Flow Diagram (RFD) focused on procedure and resource that is used in each procedure to clarify "what is need to do it", "how to do it", and "what is brought from it" considering "when to do or prepare it" according to above-mentioned education model. The approach of RFD is based on functional programing [5, 6] that units consisted by function and their input and output to improve independency of each procedure considering the application of schedule management for the collaboration in group work and the information systems. However, RFD considers the objects that function is executed on it.

The objects for RFD consist of Resource, Tool, and Task shown in Fig. 2. Task can be considered as function in functional programming thus the task has definition of input and
Fig. 2 Object for Resource Flow Diagram

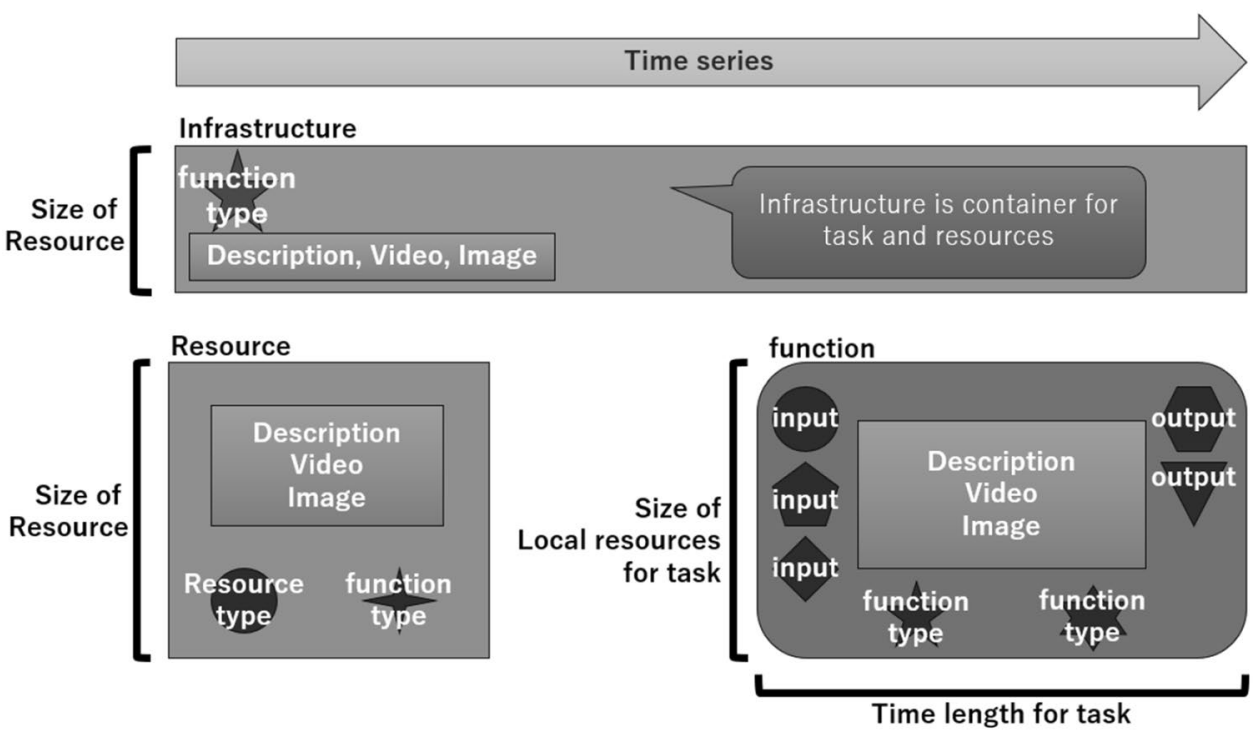


output by Resource. Resource is object used in Task such as the variable in standard programing language but it can be considered as function that store some value according to functional programming theory. The third object of RFD is Tool. Tool is function that takes the function instance as input. Tool has definition of function type that is required in task execution. Note that resource also has function type to store the resource instance such as the refrigerator for food material in cooking and the main memory for variable in programming.

The visualization of RFD is based on sequence diagram in UML [3] but RFD objects are present by graphical object and movie shown in Fig. 2 and RFD is strict to resource management in time line shown in Fig. 3. The resource type and the function type are defined by graphical icon correspond to each type. Moreover, description is presented as graphical image or video. The size of RFD object should be written by the horizontal size for activated duration and the vertical size for resource occupancy. However, resource occupancy is not considered in this experiment for simplification in student understanding.

The procedure of Tasks is written as sequence diagram with swim lines of tools. The resource and the task are arranged in tool and resource and task is connected by the line corresponding to procedure. Function type of tools and arranged resource and task should be matched. In the connection of resource and task, resource type should be matched in each other. From this type visualization, user can be thinking about event modeling with trial and error by intuitively operation.

\section{Experiment}

\subsection{Demonstration experiment method}

As the prior experiment before system development, we applied proposed education framework to the demonstrative lecture to verify the understanding effectiveness. In this experiment, the cooking video is used for modeling application. The cooking is very similar to system development and programming such that is strict to order of procedure and require resource and time management.

Table 1 shows the timetable of demonstration experiment. In this demonstration experiment, the preparation and the classroom are taken continuously in $90 \mathrm{~min}$ for evaluation. In the lecture for this demonstrative lecture, the usage of RFD is only explained before exercises. Students are consisted by $3 r d$ and 4 th degree of undergraduate. Majority of student subjects have the knowledge of system development and programming and do not cook daily.

For exercise, we develop the problem documents consisting of cooking videos and the text written about the goal of exercise. The goal of this exercise is creation of cooking procedure for given food from the combination of cooking method shown in cooking videos of 3 different foods. For the description of cooking procedure, RFD is used. The RFD of cooking video is presented to students with problem documents. Students refer the online problem documents and offline paper documents of RFDs to solve the problems. Figure 4 is overview of RFD construction of this exercise. This paper RFDs can be used to the parts of RFD for given
Fig. 3 Example of Resource Flow Diagram
How to write a Resource Flow Diagram Sample

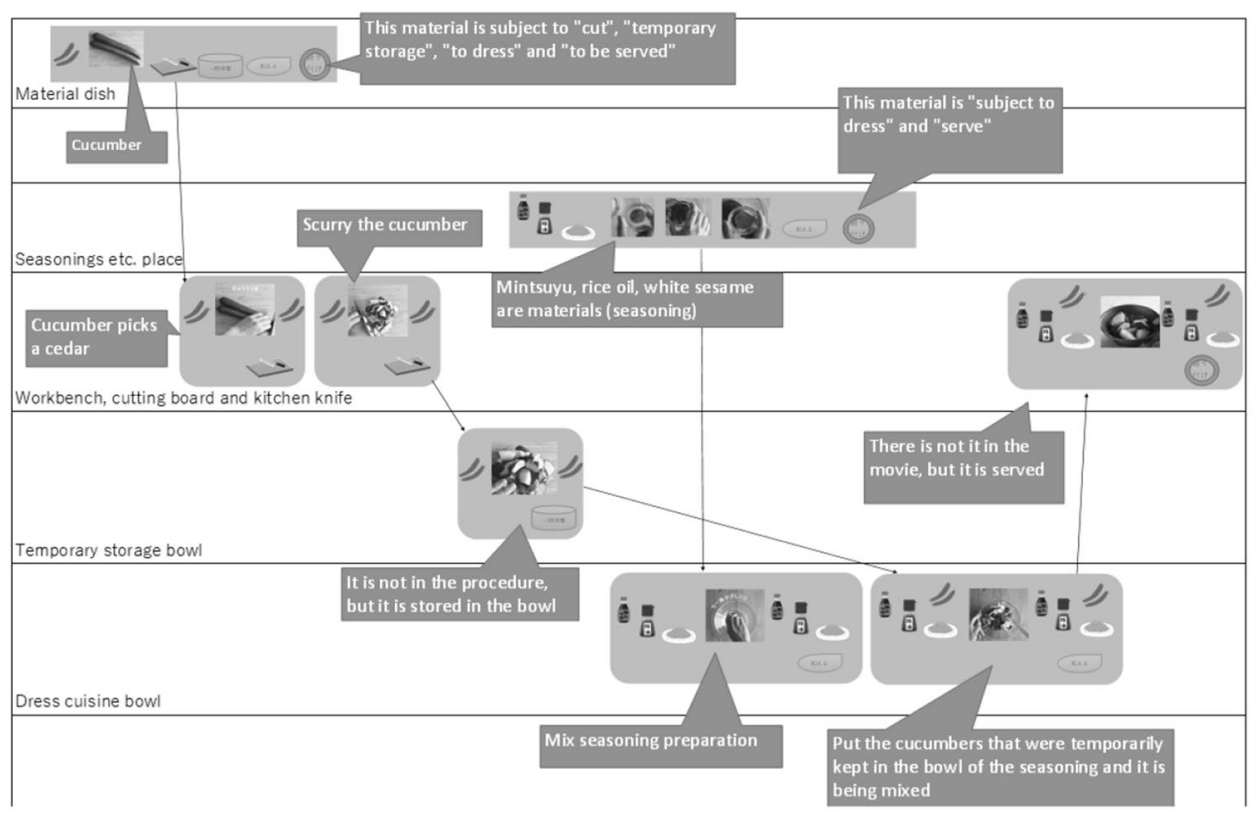


Table 1 Timetable of demonstration experiment

\begin{tabular}{lll}
\hline Start time & Program & Contents \\
\hline 18:15 & Introduction & Explain the purpose of the exercise and the overall flow \\
$18: 18$ & Lecture & How to write RFD, function, resources, infrastructure \\
$18: 28$ & Exercise 1 & Looking at three dish recipe videos and making a flow of different recipes \\
$18: 58$ & Explanation of exercise 1 & Explanation of the points to be focused on exercise 1 based on the answer exam- \\
& & ple and extracted from the movie \\
$19: 03$ & Exercise 2 & Looking at three dish recipe videos and making a flow of different recipe recipes \\
$19: 33$ & Questionnaire & Questionnaire \\
\hline
\end{tabular}

Fig. 4 Overview of RFD construction in exercise

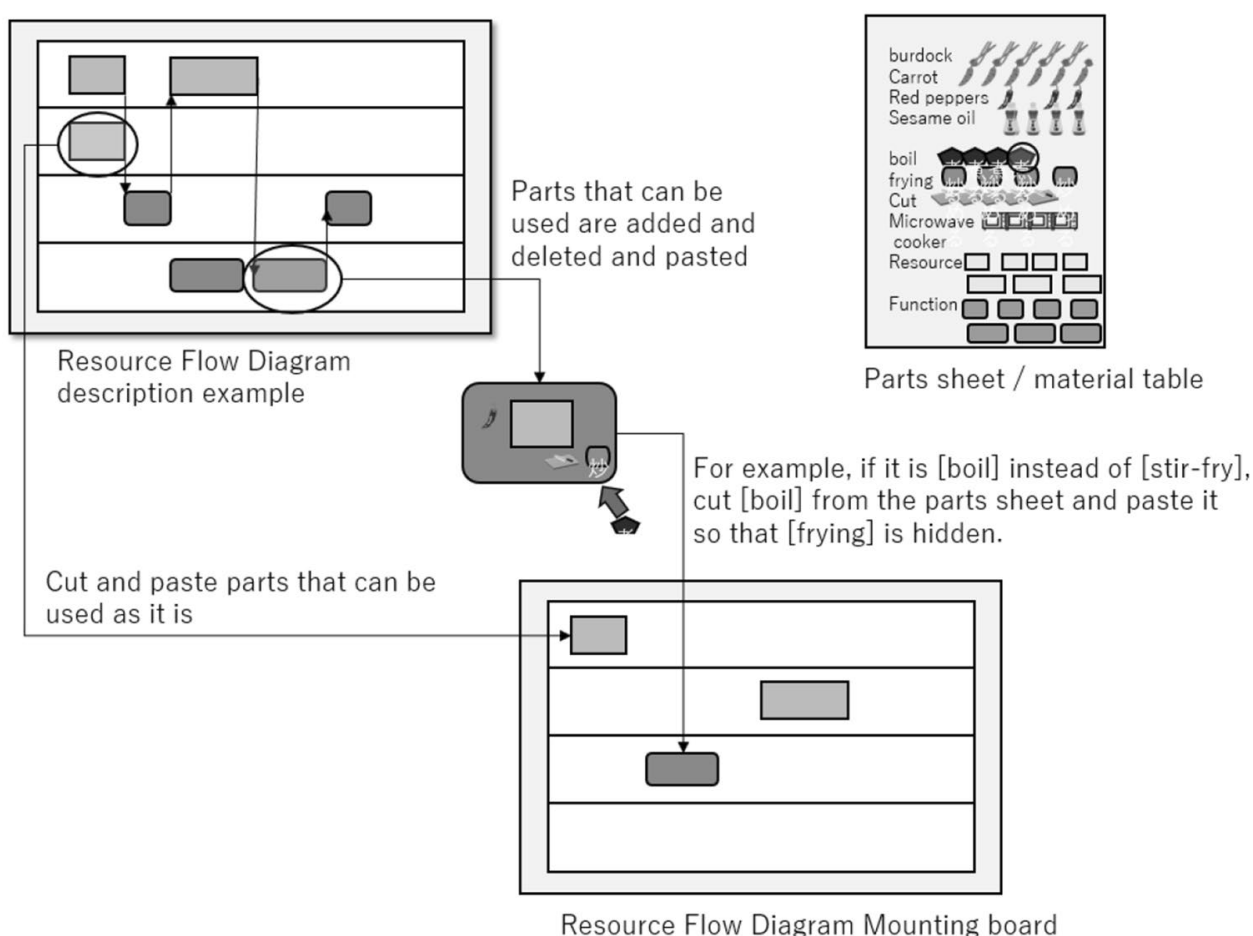

Resource Flow Diagram Mounting board food as goal combined with individual parts of Function, Resource, and Tool.

In the exercise 1, Nikumaki (meat roll up) of burdock is used for subject. Nikumaki is major home cooking in Japanese. For thinking about procedure of Nikumaki of burdock, following three cooking videos and RFDs are presented. Kinpira is a Japanese style stir frying that uses the root vegetables. Student can know the common procedures of Nikumaki and pretreatment of burdock from these videos.

- Nikumaki of asparagus

- Nikumaki of okra

- Kinpira of burdock and carrot.

After the exercise 1, thinking process using RFD in this problem was explained to understand the application of RFD for problem solving.
In the exercise 2, Oyakodon (Chicken and Egg Bowl) is used for subject. Oyakodon is major food style of rice in bowl that uses chicken and eggs. For thinking about procedure of Oyakodon, following three cooking video and RFDs are presented similar to exercise 1. Student can know the procedures of egg-bound soup and cooking method of chicken from these videos.

- Soup of leek and chicken

- Egg-bound soup with Tofu

- Tonkatu (Cutlet and Egg Bowl)

After the exercise 2, student verifies own RFD with cooking video of Oyakodon without public explanation. From the result of questionnaires after exercise 2, we consider the effectiveness of our method. 


\subsection{Result of demonstration experiment}

In the evaluation of the demonstration experiment this time, in exercise 1, we considered that the time from learning to creation of RFD was short, and we also evaluated the total score of exercises 1 and 2 or the score increment between exercises 1 and 2 .

Evaluation A was assumed to have a total score higher than 120 points. More than 120 points of evaluation A exceeds the acceptance standard 60 points on average. Evaluation B was set to a total score of 50 points or more or an increment of 30 points or more. Evaluate 30 points increment clearly understood. Evaluation $\mathrm{C}$ is a person who does not reach evaluation $\mathrm{B}$.

In this classification, class A is 2 students, class B is 9 students and class $\mathrm{C}$ is 7 students. The class $\mathrm{C}$ is considered as members who are not able to understand about problem solving in this lecture or have less motivation. On the other hand, members of class B got higher grading in exercise 2. This result shows the intuitiveness of RFD (Table 2).

\subsection{Questionnaire}

After the exercise 2, 17 valid answers are yielded from some anonymous questionnaires. The questionnaire items are shown in Fig. 5. The questionnaires consist of groups that ask about Q.A. own ability improvement, Q.B.

Table 2 Results of pre-test and post-test

\begin{tabular}{lrlrrl}
\hline Students & Exercise1 & Exercise2 & Total & Gain & Class \\
\hline Student1 & 20 & 70 & 90 & 50 & B \\
Student2 & 5 & 20 & 25 & 15 & C \\
Student3 & 10 & 80 & 90 & 70 & B \\
Student4 & 0 & 15 & 15 & 15 & C \\
Student5 & 50 & 75 & 125 & 25 & A \\
Student6 & 50 & 55 & 105 & 5 & B \\
Student7 & 10 & 10 & 20 & 0 & C \\
Student8 & 5 & 25 & 30 & 20 & C \\
Student9 & 50 & 60 & 110 & 10 & B \\
Student10 & 10 & 50 & 60 & 40 & B \\
Student11 & 25 & 60 & 85 & 35 & B \\
Student12 & 50 & 50 & 100 & 0 & B \\
Student13 & 10 & 10 & 20 & 0 & C \\
Student14 & 0 & 30 & 30 & 30 & B \\
Student15 & 15 & 15 & 30 & 0 & C \\
Student16 & 70 & 80 & 150 & 20 & A \\
Student17 & 10 & 80 & 90 & 70 & B \\
Student18 & 5 & 15 & 20 & 10 & C \\
\hline
\end{tabular}

effectiveness of RFD, Q.C. student experiments, and Q.D free comment.

\subsection{Results of Q.A. for ability improvement}

Figure 6 is result of questionnaires Q.A for own ability improvement. The answer options of Q.A questionnaires are "strongly agree", "agree a little", "Neither agree nor disagree", "disagree a little" and "strongly disagree". The answers are graded as 5 for "strongly agree" to 1 for "strongly disagree".

The median of answer grade is 4; therefore, the most student felt own ability improvement through this exercise. Specially, Q.A-3 of questionnaire about application gave higher average. This result shows effectiveness of RFD as support tool for problem solving.

\subsection{Results of Q B. for RFD}

Figure 7 shows grading result of questionnaires Q.B about RFD. The answers are converted into 5 to 1 same as Q.A; however, grading of Q.B-5 to 7 are inverted because it asks negative things about RFD.

The result of Q.B-1 to 4 about RFD representation capability is very high average grades. This result shows high intuitive representation ability of RFD. On the other hand, most students felt difficulty to defining RFD parts such as resource and tools. This difficulty is related to difficulty of problem solving thus RFD are considered to be good training tools for problem solving.

\subsection{Results of Q.C. for student profiles}

Figure 8 shows result of Q.C. for student profiles. Most questionnaires ask "yes" or "no" about student experiment related to system development and group works. Figure 9 shows result of Q.C-9 asks frequency of cooking by own self that is consisted by "no cooking by myself", " 1 or 2 days a week", "3-5 days a week", "everyday". These options are graded as 1 to 4 .

Most students take the lectures related to system development and programming. On the other hand, they have not experience about job work for it. In addition, the frequency of cooking is overall low. Therefore, it is considered that RFD based on UML is easy to understand for subject students and cooking is not intuitive as subject of exercise.

\subsection{Results Q.D of comments}

In the expression of questionnaire Q.D, the comment space was divided into positive and negative. The majority of positive comments pointed out high effectiveness of RFD as support tool for event modeling on problem solving and 
Fig. 5 List of questionnaires

Fig. 6 Results of Q.A. for ability improvement
Q.A [Questionnaires about Improvement of Problem-Solving Ability]

Please select one to each question about your own ability improvement through this exercise.

Q.A-1. How much did your ability of procedures finding improve?

Q.A-2. How much did your ability of necessary elements (resources and tools) finding improve?

Q.A-3. How much did your ability of application from another solutions finding improve?

Q.B [Questionnaires about RFD]

Please select one to each question about RFD used in the exercise.

Q.B-1. RFD can be used to clarify the flow of the procedure

Q.B-2. RFD can be used to clarify the resource type

Q.B-3. RFD can be used to clarify the duration of the resource's existence

Q.B-4. RFD can be used to clarify the operational status of the infrastructure

Q.B-5. Definition of the procedure is difficult

Q.B-6. Definition of the resources is difficult

Q.B-7. Definition of the tools is difficult

Q.B-8. RFD can be applied to programming

Q.B-9. RFD can be applied to the design of server system etc.

Q.C [Questionnaires about Student Profile]

Please select one to each question about your experience.

Q.C-1. Course related to programming

Q.C-2. Course related to communication network

Q.C-3. Course related to system design

Q.C-4. Development of own software

Q.C-5. Development of own server system

Q.C-6. Part-time job

Q.C-7. Job other than part-time

Q.C-8. Engineer of system development or programmer.

Q.C-9. Frequency of cooking by oneself

Q.D [Comment]

Free response.

Q.D-1. Please tell us your feeling that this learning model is good.

Q.D-2. Please tell us your feeling that this learning model is not good.

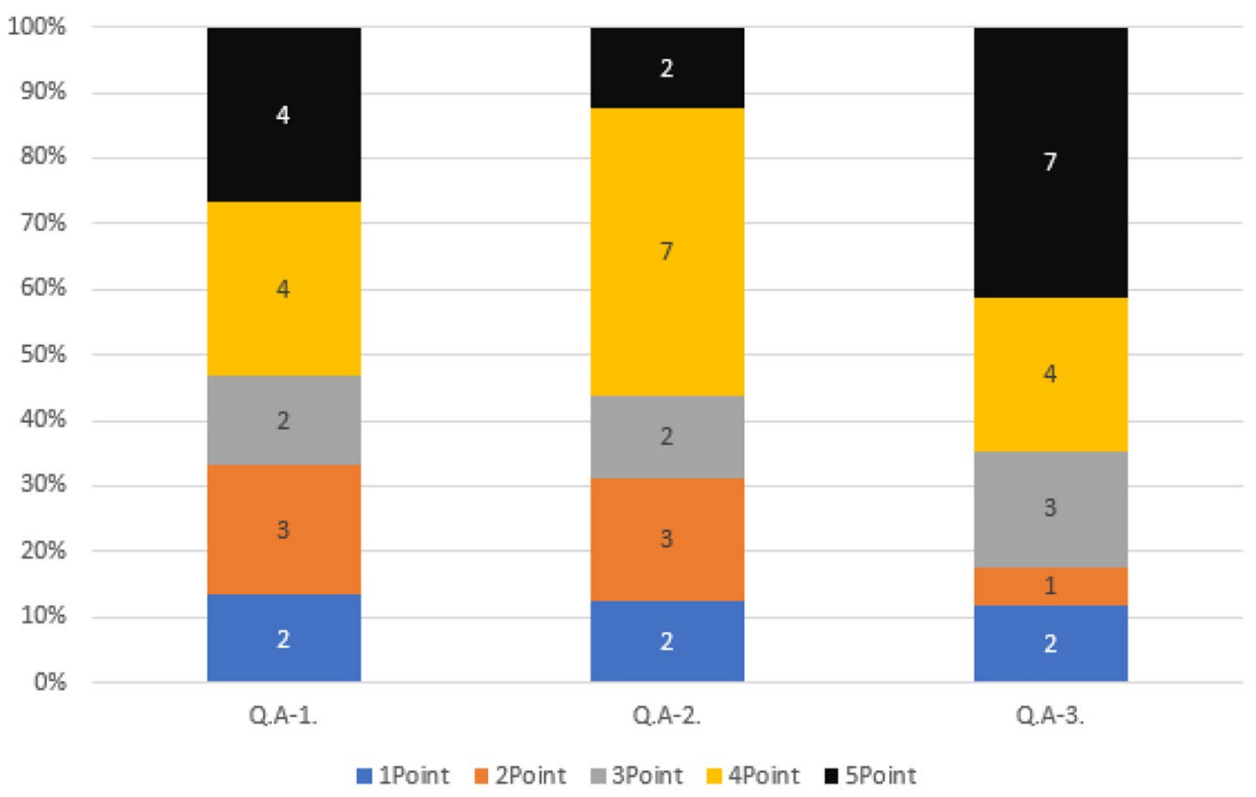


Fig. 7 Results of Q B. for RFD

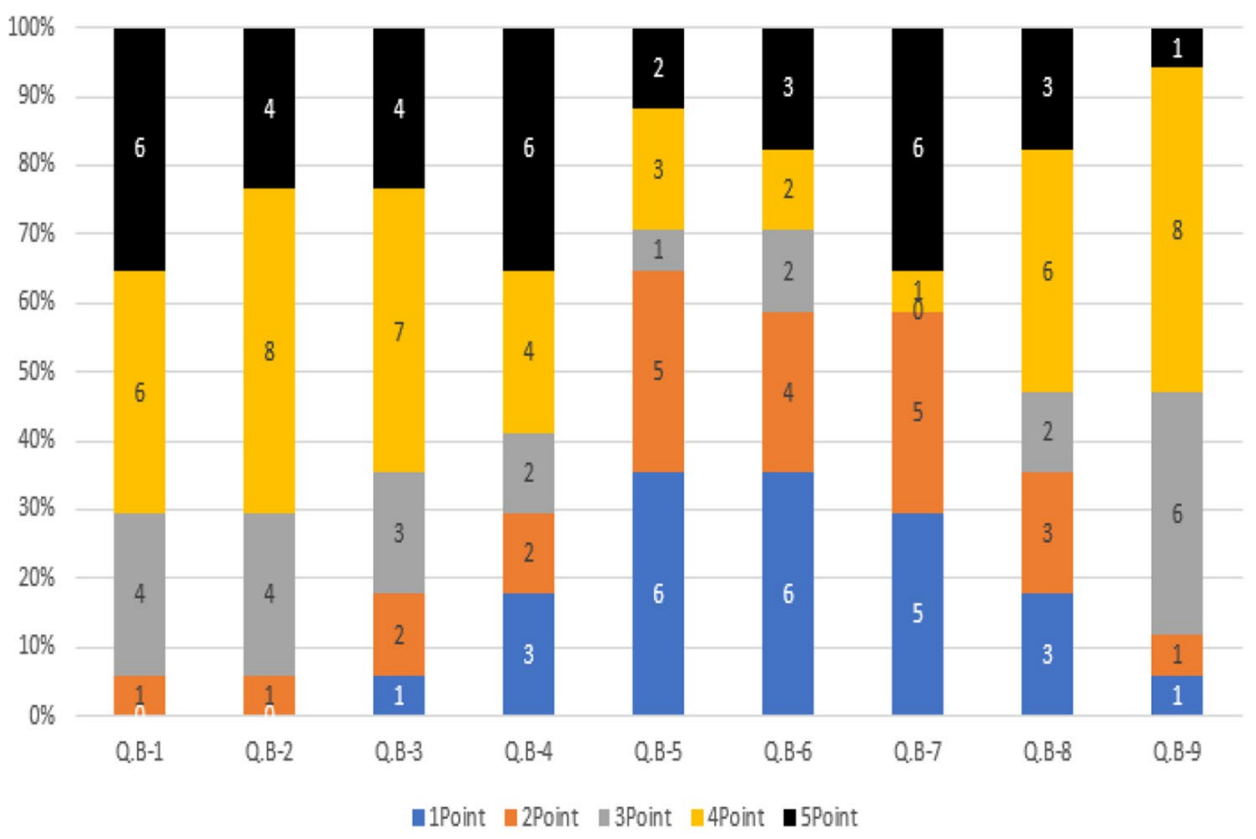

Fig. 8 Results of Q.C.1-8 for student profiles

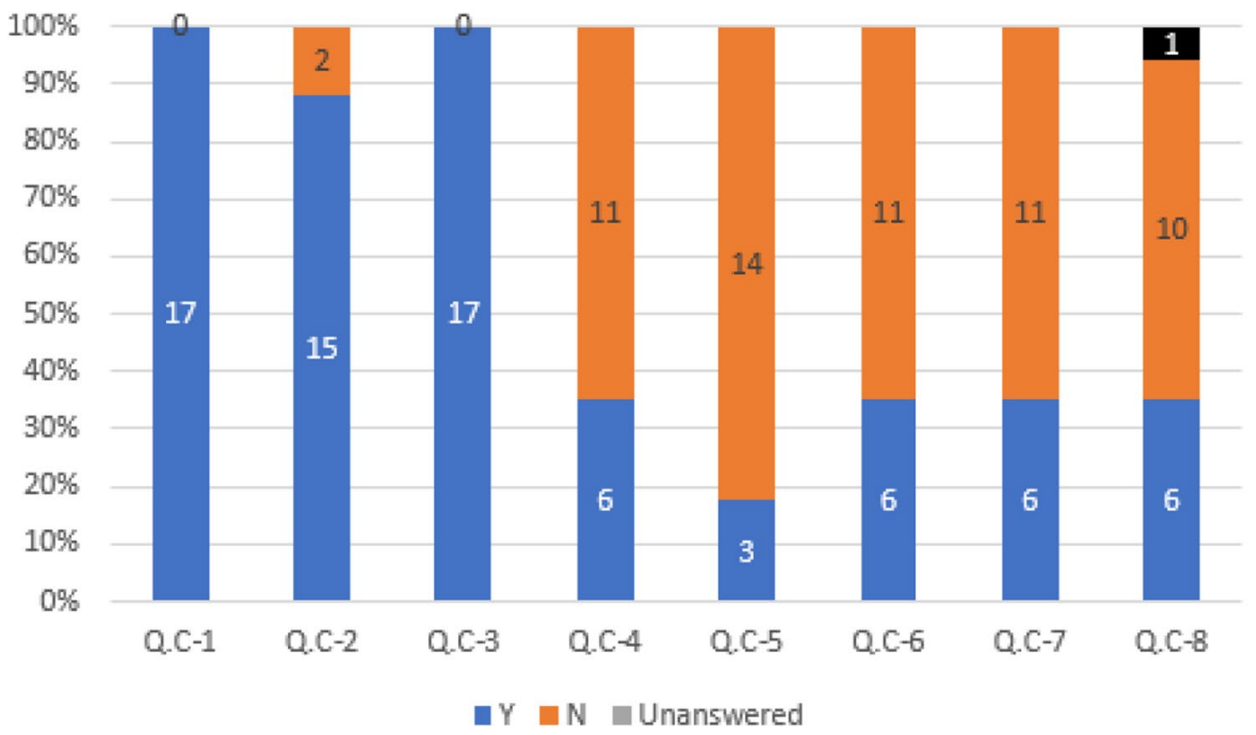

high intuitiveness by trial and error with their hand. For this result, high intuitiveness is very effective to train problem solving with practice in elementary education. We plan to develop information systems for achievement of problemsolving education with trial and error using RFD.

The majority of negative comments pointed out the lack of exercise time and time length of hand working for RFD. However, it can be resolved by support system for RFD construction in the future.

\subsection{Correlation between questionnaires}

When Spearman rank correlation of each questionnaire is obtained, Q.A-1 and Q.A-3 and Q.A-1 and Q.B-1 show high correlation coefficient 0.83 . This result shows that RFD is effective for solving problems from a combination of knowledge on solving similar problems. 
Fig. 9 Results of Q.C.9 for student profiles

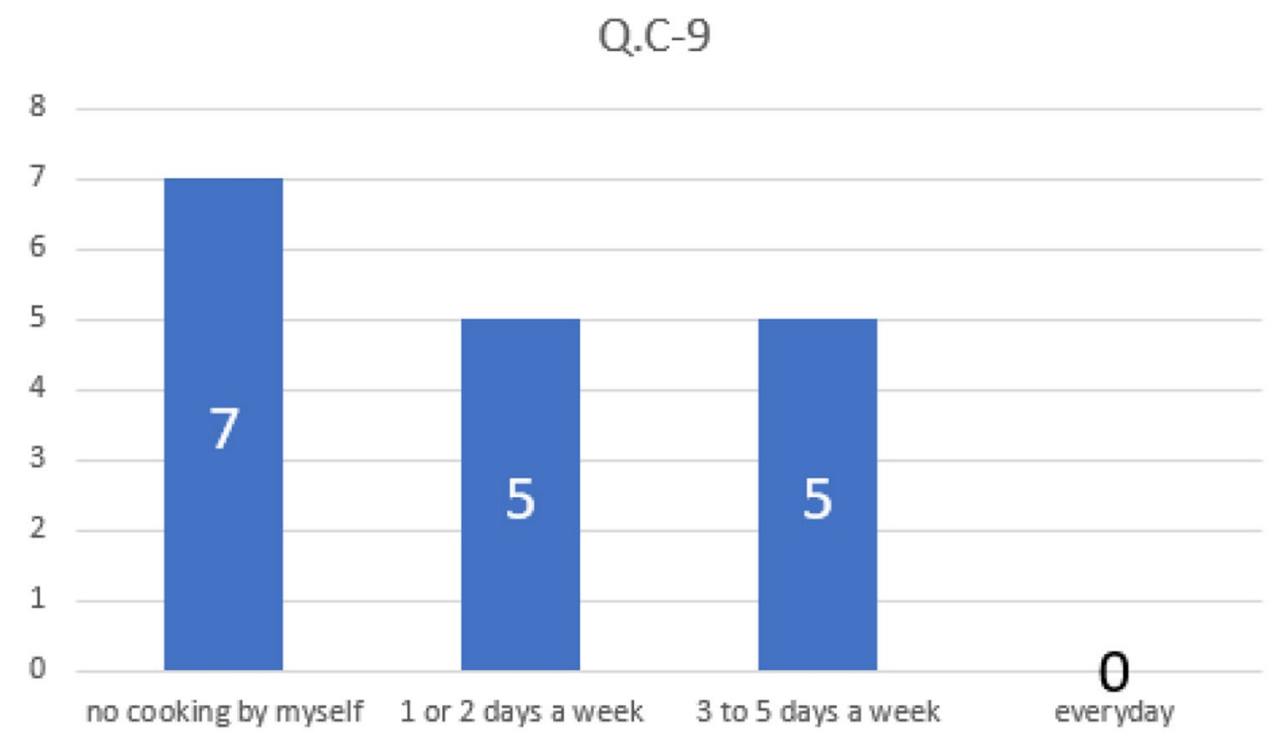

\section{Conclusion}

In this paper, we proposed RFD to achieve our problem-solving education framework based on the modeling from video. The approach of our problem-solving education focuses on the event modeling from non-verbalized complex problem such as video. In this experiment, we investigated proposed framework in demonstrative lecture of cooking that has similar characteristics to system development and programming. From this experiment, good questionnaire results were obtained that the intuitive representation of RFD and its potential application to information system design are high. Considering that the less language dependency of our education framework occurs intuitively, it is expected to be effective to elementary education of problem solving for highly informed society.

From the negative result of this experiment, RFD and exercise using RFD require system support to facilitate trial and error. We plan to develop the RFD editor on computer and offline education system like block toys to reduce the complication in the future.

Acknowledgements This research was supported by JSPS KAKENHI Grant number 15K01086.

Open Access This article is distributed under the terms of the Creative Commons Attribution 4.0 International License (http://creativeco mmons.org/licenses/by/4.0/), which permits unrestricted use, distribution, and reproduction in any medium, provided you give appropriate credit to the original author(s) and the source, provide a link to the Creative Commons license, and indicate if changes were made.

\section{References}

1. Bernardi S, Donatelli S, Merseguer J (2002) From UML sequence diagrams and statecharts to analysable petri net models. In: Proceedings of the 3rd international workshop on Software and performance. ACM, New York, pp. 35-45

2. Petre M (2013) UML in practice. In: Proceedings of the 2013 international conference on software engineering. IEEE Press, Piscataway, pp. 722-731

3. Nunohiro E, Kishimoto Y, Yamaguchi T, Ohshiro M, Tsukuta T (2017) Development of practice problems generating function in PPL system. In: Proceedings of the 22nd international symposium on artificial life and robotics

4. Bird RS, Wadler PL (1988) Functional programming. Prentice Hall, Upper Saddle River

5. Etzion O, Niblett P, Luckham DC (2011) Event processing in action. Manning, Greenwich

6. Miles R, Hamilton K (2005) Learning UML 2.0: a pragmatic introduction to UML. O'Reilly Media, pp 56

Publisher's Note Springer Nature remains neutral with regard to jurisdictional claims in published maps and institutional affiliations. 